Check for updates

Cite this: RSC Adv., 2019, 9, 3162

Received 16th November 2018

Accepted 9th January 2019

DOI: 10.1039/c8ra09443h

rsc.li/rsc-advances

\title{
Mechanism of surface treatments on carbon nanotube transparent conductive films by three different reagents
}

\author{
Ze-Zeng Gu, Song-Lin Jia, Guangfen Li, (D) * Chunqing Li, Yan-Qi Wu \\ and Hong-Zhang Geng (D)
}

\begin{abstract}
Transparent conductive films (TCFs) were fabricated via a spray-coating method with a solution prepared by dispersing single-walled carbon nanotubes (SWCNTs) in deionized water with sodium dodecylbenzene sulfonate (SDBS) as surfactant. We explored the mechanism of $\mathrm{HNO}_{3}$ treatment by treating TCFs with different reagents. After being treated with different concentrations of reagents by $\mathrm{HNO}_{3}, \mathrm{HCl}$, and $\mathrm{NaNO}_{3}$ to lower the sheet resistance of TCFs, the properties of TCFs were further characterized by a UV-VIS spectrophotometer, a four-point probe method, atom force microscopy, X-ray photoelectron spectroscopy, and Raman spectroscopy. In this study, we conclude that the $\mathrm{HNO}_{3}$ treatment results in a decrease in the sheet resistance of the TCFs due to the combined effect of acidity and oxidizability. The strong interaction of the strong acidity and strong oxidizing property of $\mathrm{HNO}_{3}$ causes the SDBS to be removed. To further improve the film conductivity of the TCFs, the experimental conditions of the $\mathrm{HNO}_{3}$ treatment were optimized.
\end{abstract}

\section{Introduction}

Due to the rapid development of electronic equipment, the demand for transparent conductive films (TCFs) has become more and more urgent. More stringent requirements on the physical and chemical properties of conducting materials have to be put forward. To this goal, a lot of research regarding flexible TCFs has been carried out incorporating different conductive nanomaterials such as carbon nanomaterials, ${ }^{\mathbf{1 , 2}}$ metallic nanowires, ${ }^{3}$ nanoparticles, ${ }^{4}$ and conductive polymers. ${ }^{5}$

Carbon nanotubes (CNTs) have attracted a lot of research attention due to their specific structure and excellent mechanical, optical and electrical properties, ${ }^{6,7}$ and thus have been used in diverse fields as transistors, ${ }^{8}$ storage devices, ${ }^{9}$ and optoelectronic devices. ${ }^{10} \mathrm{CNT}$ incorporated transparent conductive films (CNT-TCFs) can be used in a flexible electroluminescent device because CNTs can also be introduced as a hole injection buffer layer on ITO to realize a high brightness organic light emitting diode (OLED). ${ }^{11,12}$ CNT-TCFs can be prepared by direct growth, solution deposition, spray coating, and vacuum filtration methods. ${ }^{13}$ Among them, spray coating is a commonly used preparation method, which has the advantage of low cost, simplicity, easy realization of large scale films and uniform film formation. ${ }^{\mathbf{1 4}}$ Because of the large specific surface area in carbon

State Key Laboratory of Separation Membranes and Membrane Processes, Key Laboratory of Advanced Fibers and Energy Storage, School of Material Science and Engineering, Tianjin Polytechnic University, Tianjin, 300387, China. E-mail: liguangfen@tjpu.edu.cn; Fax: +86-22-83955055; Tel: +86-22-83955814 nanotubes, a strong van der Waals force among the nanotubes induces CNT entanglement and results in the formation of agglomerates. To solve this problem, a suitable surfactant is required in the preparation process of CNT dispersion. However, the tiny amount of surfactant present in the TCFs affects the conductivity due to its inherent insulating properties. $^{15,16}$ Therefore, it is necessary to remove the surfactant by post-treatment in order to improve the conductivity of the CNTTCFs. There are several post-processing methods for improving the conductivity of the CNT-TCFs without affecting the transmittance, such as immersion in $\mathrm{HNO}_{3}, \mathrm{H}_{2} \mathrm{SO}_{4}$ or $\mathrm{SOCl}_{2}$, and $p$ type and $n$-type doping of the CNTs. ${ }^{17-21}$ Compared to these methods, a very effective treatment of using $\mathrm{HNO}_{3}$ is suggested. The purpose of this treatment is to remove the surfactants used for dispersing CNTs in solution and create a denser CNT film. ${ }^{21}$ However, it does not further explain what properties of $\mathrm{HNO}_{3}$ reduce the sheet resistance of CNT-TCFs. The improvement in film transmittance and sheet resistance was attributed to the acidic properties instead of an oxidation effect. Therefore, in this study, we further explored the mechanism of $\mathrm{HNO}_{3}$ treatment by treating CNT-TCFs with different reagents.

In this work, we prepared CNT-TCFs by spraying CNT solution on a polyethylene terephthalate (PET) substrate. The effects of different kinds of reagents, the concentrations of posttreatment reagents, and post-treating time on the electrical and optical properties of CNT-TCFs were investigated by versatile methods. In order to understand the effect of posttreatment by $\mathrm{HNO}_{3}$, three reagents $\left(\mathrm{HNO}_{3}, \mathrm{HCl}\right.$, and $\left.\mathrm{NaNO}_{3}\right)$ were selected to post-treat the CNT-TCFs for comparison. $\mathrm{HNO}_{3}$ 
contains $\mathrm{H}^{+}$and $\mathrm{NO}_{3}{ }^{-}$, while $\mathrm{NaNO}_{3}$ contains $\mathrm{NO}_{3}{ }^{-}$and $\mathrm{HCl}$ contains $\mathrm{H}^{+}$. These two reagents were chosen as a comparative study to understand the mechanism of $\mathrm{HNO}_{3}$ treatment, and to explore which kinds of ions play an effective role to reduce the resistance of CNT-TCFs.

\section{Experimental section}

2.1 Preparation of a single-walled carbon nanotube solution

$0.02 \mathrm{~g}$ single-walled carbon nanotubes (SWCNTs) with a purity of $90 \%$ were dispersed into $20 \mathrm{ml}$ water by using $0.2 \mathrm{~g}$ sodium dodecylbenzene sulfonate (SDBS) as the dispersant. A concentration of $1 \mathrm{mg} \mathrm{ml}^{-1}$ of SWCNT solution was prepared and then ultrasonicated for $40 \mathrm{~min}$. The solution was further ultrasonicated at $120 \mathrm{w}$ for another $35 \mathrm{~min}$ in order to obtain a better dispersion of CNTs. The resulting homogeneous SWCNT dispersion was centrifuged at $8000 \mathrm{rpm}$ for $15 \mathrm{~min}$, and the supernatant was transferred to a clean beaker. ${ }^{15,22}$

\subsection{Preparation and characterization of the carbon nanotube conductive films}

A PET film was cut to $5 \mathrm{~cm} \times 5 \mathrm{~cm}$ in size. Then the PET film was ultrasonicated in distilled water for one hour and then in ethanol for one hour. Subsequently, the PET film was dried with an air dryer. CNT-TCFs were prepared by spraying the SWCNT solution on the cleaned PET substrates. The temperature of the PET substrate was maintained at about $100{ }^{\circ} \mathrm{C}$ for the solution to dry quickly. The dried CNT-TCFs were then immersed in distilled water for $20 \mathrm{~min}$ and then washed with distilled water 4 times. The effects of the three different reagents ( $\mathrm{HCl}(38 \mathrm{wt} \%)$, $\mathrm{HNO}_{3}(68 \mathrm{wt} \%)$, and a saturated solution of $\left.\mathrm{NaNO}_{3}\right)$, the ratio of acid to water, the post-treatment time for removing the residual surfactants on the surface resistance and transmittance of CNTTCFs were studied.

The transmittance of the CNT-TCFs was measured by a UVVIS spectrophotometer at a wavelength of $550 \mathrm{~nm}$, and the sheet resistance of the as-prepared and post-treatment CNTTCFs was obtained by using the four-point probe method (Keithley 2700 multimeter) in sequence. The morphology of the CNT-TCFs was observed using a field emission scanning electron microscope (FE-SEM, HITACHI S-4800) and an atomic force microscope (AFM, Agilent-S5500). The surface changes were detected by X-ray photoelectron spectroscopy (XPS, ELMER PHI 5600, $\mathrm{Al} \mathrm{K \alpha}$ source). Raman spectra were used to characterize the change of CNTs via a DXR Raman microscope with a laser excitation wavelength of $532 \mathrm{~nm}$.

\section{Results and discussion}

3.1 The effect of different reagents during post-treatment on sheet resistance and transmittance of the films

Sheet resistance and transmittance are found to be the most crucial factors dominating the performance of the CNT-TCFs. In order to enhance the conductivity, the CNT-TCFs were posttreated by three different reagents at the same concentration, which were $\mathrm{HNO}_{3}$ solution, $\mathrm{HCl}$ solution and a saturated solution of $\mathrm{NaNO}_{3}$, respectively. The sheet resistance and transmittance of CNT-TCFs were affected by the use of different reagents during post-treatment, as shown in Fig. 1.

In comparison with untreated CNT-TCFs, a higher sheet resistance was found for the CNT-TCFs after post-treatment by $\mathrm{NaNO}_{3}$. This suggested that the $\mathrm{NO}_{3}{ }^{-}$ion is incapable of removing the surfactant. The TCFs post-treated by a solution of either $\mathrm{HCl}$ or $\mathrm{HNO}_{3}$ show a lower sheet resistance compared with the untreated CNT-TCFs, indicating that the acidic environment favors the removal of surfactants in CNT-TCFs. Moreover, the lowest sheet resistance was found for the CNTTCFs treated with $\mathrm{HNO}_{3}$ solution, which is consistent with a previous report. ${ }^{17}$ As TCFs were placed in the $\mathrm{HNO}_{3}$ solution, the CNTs in the CNT-TCFs may undergo chemical oxidation during post-treatment by attachment of functional groups such as carboxyl groups. From this we believe that the $\mathrm{H}^{+}$in $\mathrm{HNO}_{3}$, that is the strong acidity, causes the non-conductive SDBS to be removed from the surface of the CNTs.

\subsection{The effect of diluted nitric acid during post-treatment on sheet resistance and transmittance of films}

The above results clearly demonstrate that the sheet resistance of the CNT-TCFs treated by an acidic solution of TCFs decreased significantly. To further improve the film conductivity of the TCFs, the experimental conditions with $\mathrm{HNO}_{3}$ were optimized. The effects of post-treating time (from $0,20,40$, to $60 \mathrm{~min}$ ) and the ratio of acid to water (from $4: 1,6: 1$, to $8: 1$ ) on the sheet resistance and transmittance are presented in Fig. 3. As posttreating time increases to $60 \mathrm{~min}$, the sheet resistance dramatically decreases.

As shown in Fig. 2, the sheet resistance and the transmittance decrease with $\mathrm{HNO}_{3}$ treating time and ratio of $\mathrm{HNO}_{3}$ to water simultaneously. The dramatic effect can be found for a certain $\mathrm{HNO}_{3}$ treating time. With increasing the time to $60 \mathrm{~min}$, the sheet resistance reaches the lowest desirable value of $132.2 \Omega \mathrm{sq}^{-1}$. However, the transmittance value also drops below $80 \%$. To maintain a higher transmittance, a moderate

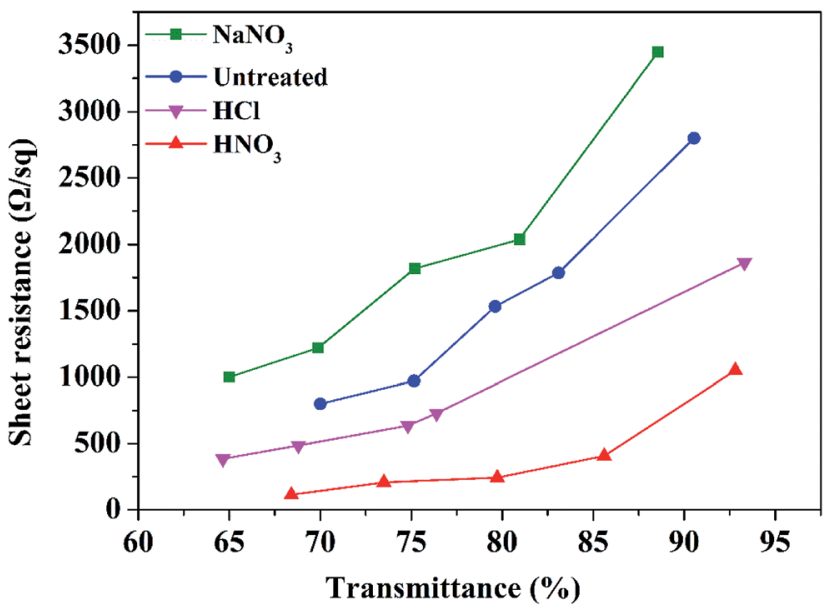

Fig. 1 The sheet resistance and transmittance of CNT-TCFs posttreated by different reagents. 

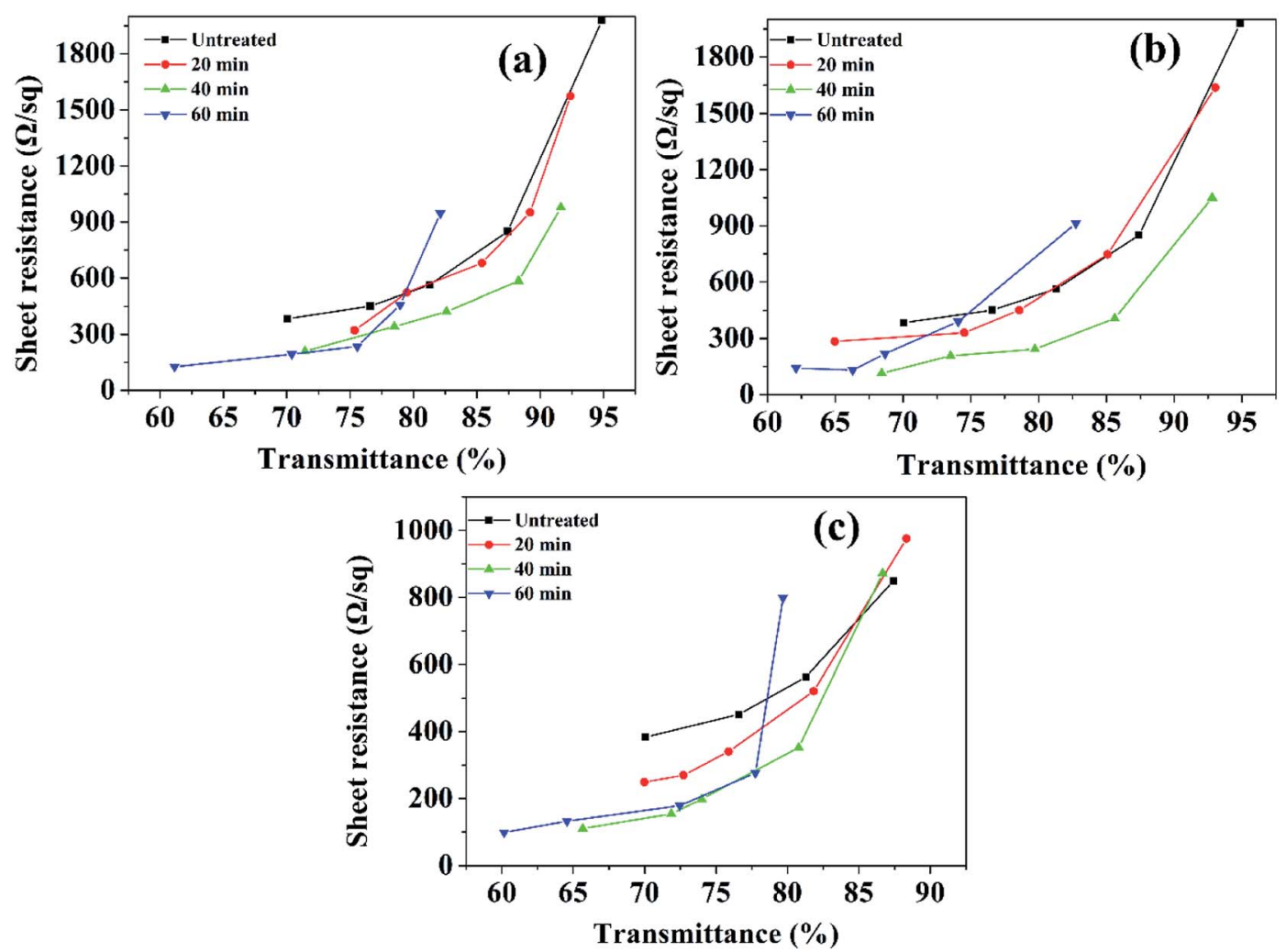

Fig. 2 The sheet resistance and transmittance of the CNT-TCFs treated by $\mathrm{HNO}_{3}$ for a time from $0,20,40$, to 60 min with different ratios of $\mathrm{HNO}_{3}$ to water from (a) $4: 1$, (b) $6: 1$, and to (c) $8: 1$.
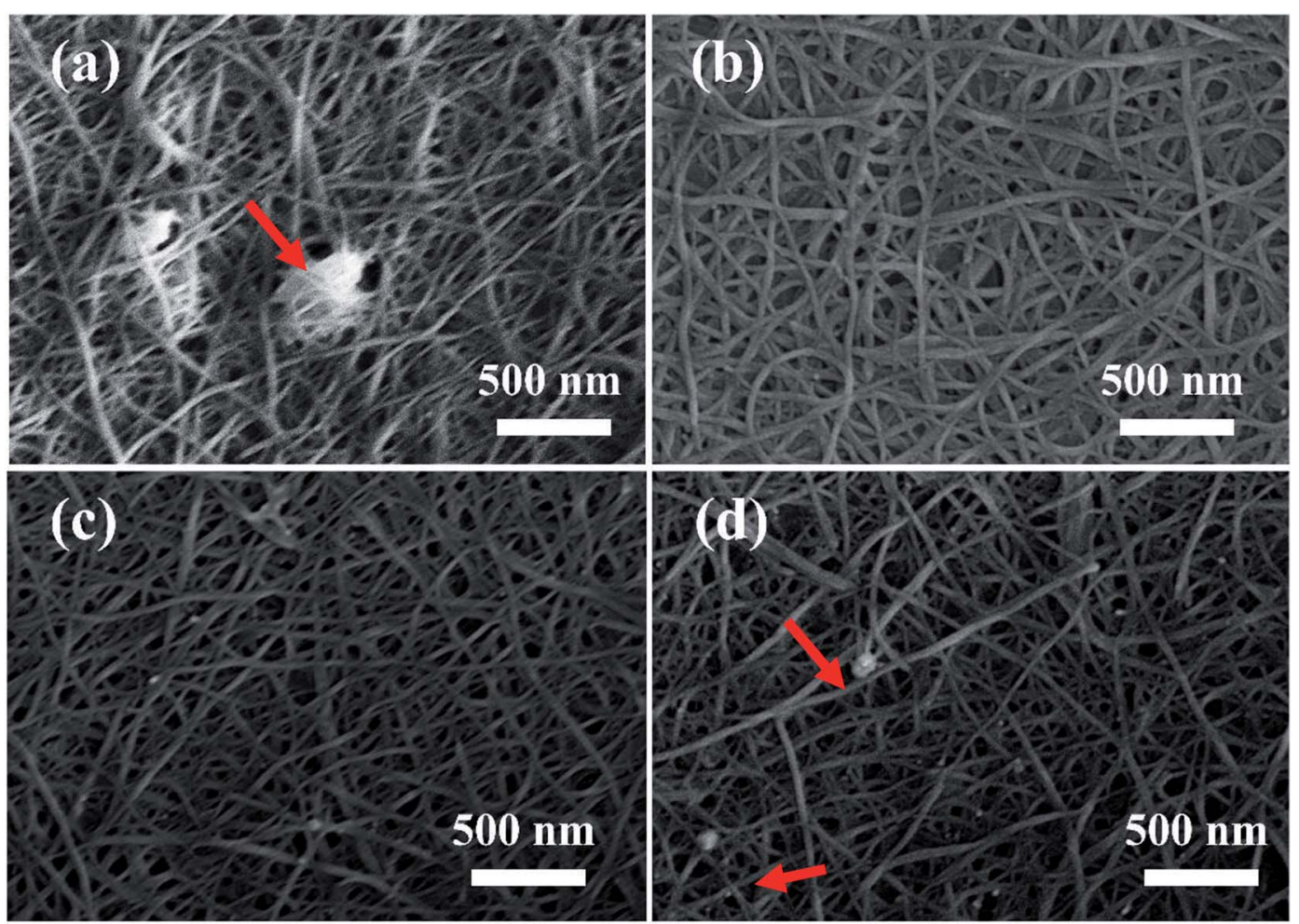

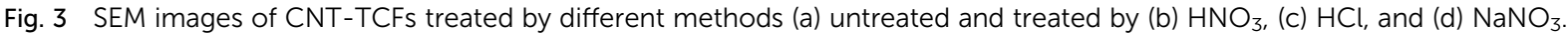


(a)

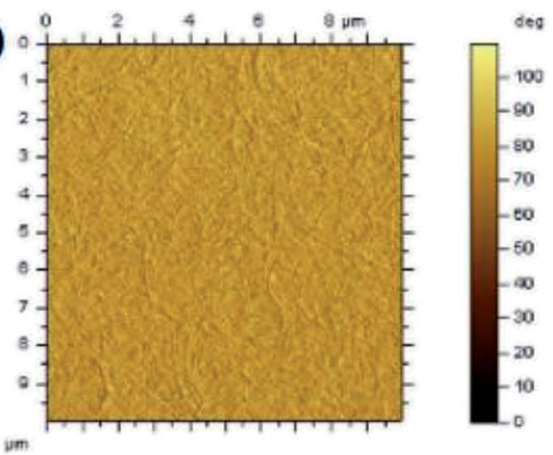

(c)

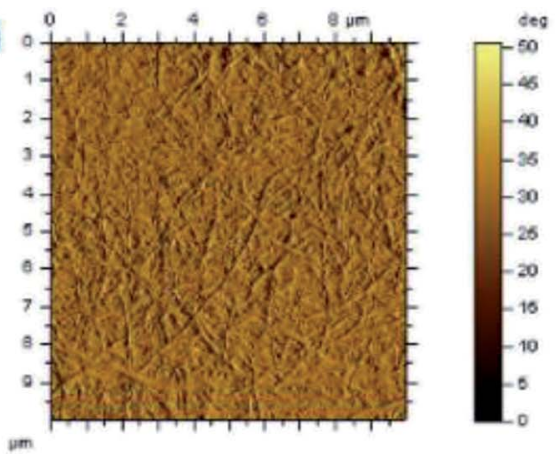

(e)
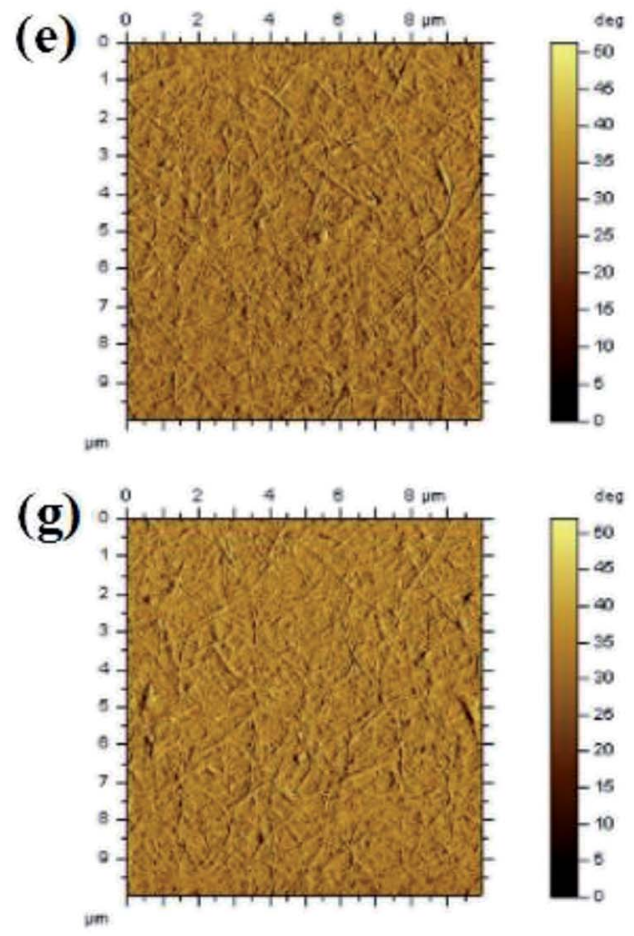
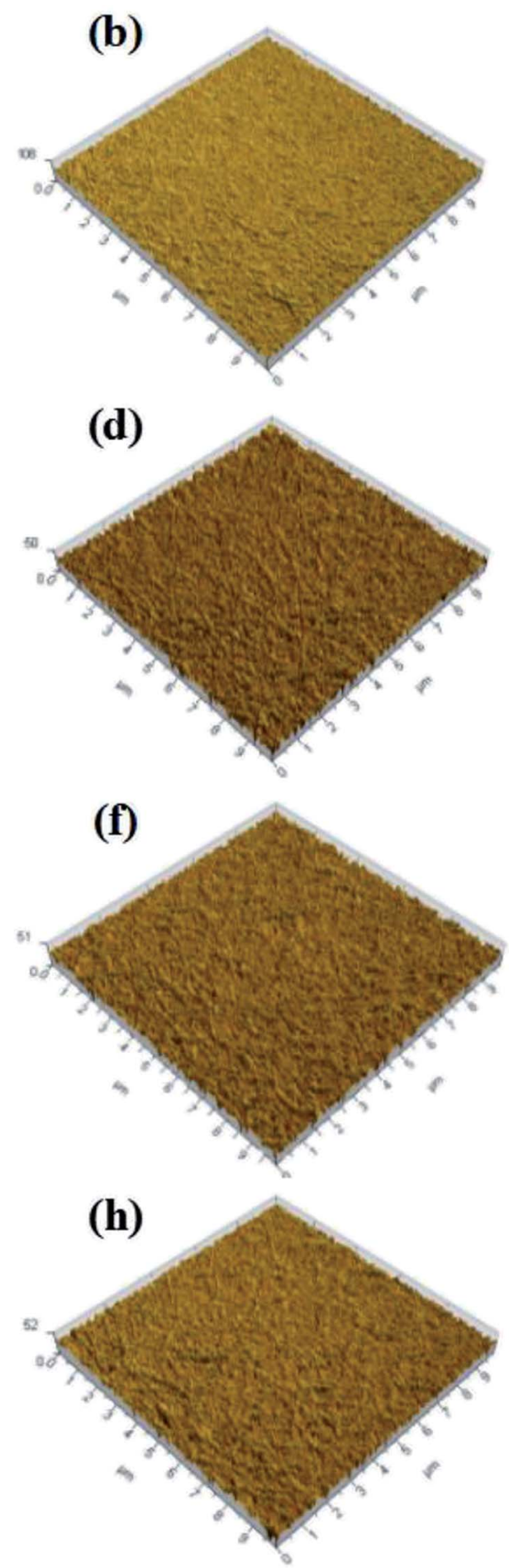

Fig. 4 AFM two and three dimensional phase images of untreated CNT-TCFs (a and b), and CNT-TCFs treated by $\mathrm{HNO}_{3}$ (c and d), $\mathrm{HCl}$ (e and f), and $\mathrm{NaNO}_{3}$ (g and h), respectively.

condition for post-treating TCFs is necessary. The results show that both parameters of sheet resistance and transmittance become acceptable, as the treating time is set as $40 \mathrm{~min}$. Fig. 2 also shows a similar effect of the ratio of acid to water on the film properties. With increasing the $\mathrm{HNO}_{3}$ content, the sheet resistance/transmittance declines/goes up simultaneously. A desirable condition was found for the ratio of acid to water as
$6: 1$ where the sheet resistance is below $500 \Omega \mathrm{sq}^{-1}$ and transmittance is about $85 \%$.

\subsection{Surface morphology of films affected by different reagents during post-treatment}

To reveal the effect of surface morphologies on the performance of CNT-TCFs, the CNT-TCFs treated by $\mathrm{HNO}_{3}, \mathrm{HCl}$ and $\mathrm{NaNO}_{3}$ 
Table 1 AFM roughness parameters of the CNT-TCFs treated by different reagents ${ }^{a}$

\begin{tabular}{lllll}
\hline & Untreated & $\begin{array}{l}\text { Treated by } \\
\mathrm{HNO}_{3}\end{array}$ & $\begin{array}{l}\text { Treated by } \\
\mathrm{HCl}\end{array}$ & $\begin{array}{l}\text { Treated by } \\
\mathrm{NaNO}_{3}\end{array}$ \\
\hline$S_{\mathrm{a}}(\mathrm{nm})$ & 28.9 & 25.5 & 26.7 & 27.5 \\
$S_{\mathrm{q}}(\mathrm{nm})$ & 33.0 & 31.0 & 31.1 & 31.5 \\
$S_{\mathrm{z}}(\mathrm{nm})$ & 89.0 & 87.3 & 90.0 & 93.3
\end{tabular}

${ }^{a} S_{\mathrm{a}}(\mathrm{nm})$ : roughness average, $S_{\mathrm{q}}(\mathrm{nm})$ : root mean square roughness, $S_{\mathrm{z}}$ (nm): ten-point height.

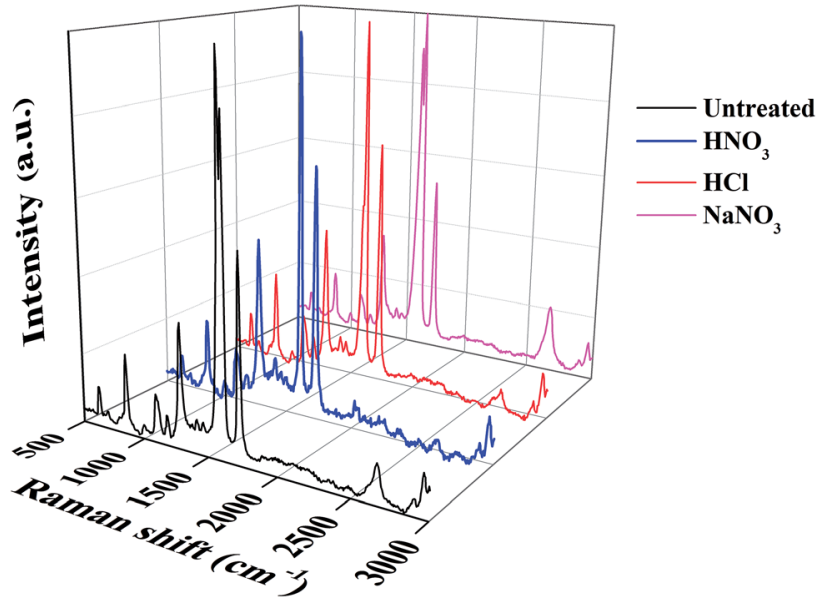

Fig. 5 Raman spectra (at $532 \mathrm{~nm}$ ) of the untreated (/D $/ I_{G} \sim 0.30$ ) CNTTCFs, and CNT-TCFs treated by $\mathrm{HNO}_{3}\left(I_{\mathrm{D}} / /_{\mathrm{G}} \sim 0.41\right), \mathrm{HCl}\left(/_{\mathrm{D}} / /_{\mathrm{G}} \sim 0.39\right)$ and $\mathrm{NaNO}_{3}\left(I_{\mathrm{D}} / I_{\mathrm{G}} \sim 0.31\right)$, respectively.

are imaged by FE-SEM and shown in Fig. 3. The CNT-TCFs without treatment and CNT-TCFs treated by a saturated solution of $\mathrm{NaNO}_{3}$ have residual SDBS inside the CNT network, which definitely impedes the film conductivity. Whereas, for the CNT-TCFs treated with two acids, little residual SDBS can be found. CNTs are homogeneously distributed on the film surface. From this we can know that the strong acidity of nitric acid removes residual SDBS.
3.4 Surface topology and roughness of CNT-TCFs treated by different reagents during post-treatment

The AFM images of the surface topology for CNT-TCFs are shown in Fig. 4 and Table 1. The root mean square roughness values of untreated CNT-TCFs and CNT-TCFs treated with $\mathrm{HNO}_{3}, \mathrm{HCl}$, and $\mathrm{NaNO}_{3}$ were $33.0 \mathrm{~nm}, 31.0 \mathrm{~nm}, 31.1 \mathrm{~nm}$, and $31.5 \mathrm{~nm}$, respectively. We assume that the SDBS particles on the CNT-TCF surface were removed during the post-treatment process. As seen from the diagram, the surface roughness of the film is reduced after nitric acid treatment. This is beneficial to its application in the transparent electrode field.

\subsection{Characterization of the CNT-TCFs by Raman spectra and XPS}

Raman spectra were carried out to characterize the change of CNTs in the CNT-TCFs with an excitation laser wavelength of $532 \mathrm{~nm}(2.33 \mathrm{eV})$. As shown in Fig. 5, the D peak near $1280 \mathrm{~cm}^{-1}$ is a reflection of the defects and disorder in CNTs whereas the G peak near $1580 \mathrm{~cm}^{-1}$ represents the order of the CNTs. ${ }^{23,24}$ The ratio of $I_{\mathrm{D}} / I_{\mathrm{G}}$ reflects the degree of disorder and defects in the sample. Here the change of $I_{\mathrm{D}} / I_{\mathrm{G}}$ can also reflect the degree of surface functionalization. ${ }^{25}$ As can be calculated from Fig. 5, the $I_{\mathrm{D}} / I_{\mathrm{G}}$ ratio of the film treated by $\mathrm{NaNO}_{3}(0.31)$ is little changed as compared to that of the untreated CNT-TCFs (0.30), indicating that the CNT surface structure has not been changed a lot by $\mathrm{NaNO}_{3}$. An increase in the $I_{\mathrm{D}} / I_{\mathrm{G}}$ ratio was found for the CNTTCFs treated by acid solution, and the $I_{\mathrm{D}} / I_{\mathrm{G}}$ value for $\mathrm{HNO}_{3}$ treated CNT-TCFs (0.41) is higher than that for $\mathrm{HCl}$ treated CNTTCFs (0.39). This suggests that chemical oxidation might actually occur and result in detachment of the surfactant from the CNT surface, and eventually lower the sheet resistance. ${ }^{26}$

XPS analysis was used to provide information about element contents present in the CNT-TCFs after being treated by three different reagents, as shown in Fig. 6. As shown in Fig. 6a, a similar height of the $\mathrm{S} 2 \mathrm{p}$ peaks appears in the three samples of untreated TCFs and TCFs treated by $\mathrm{HCl}$ and $\mathrm{NaNO}_{3}$. The sulfur content of the CNT-TCFs treated with $\mathrm{HCl}$ and $\mathrm{NaNO}_{3}$ did not decrease significantly, indicating that SDBS still has some residues. The weakest $\mathrm{S} 2 \mathrm{p}$ signal was found for the sample after
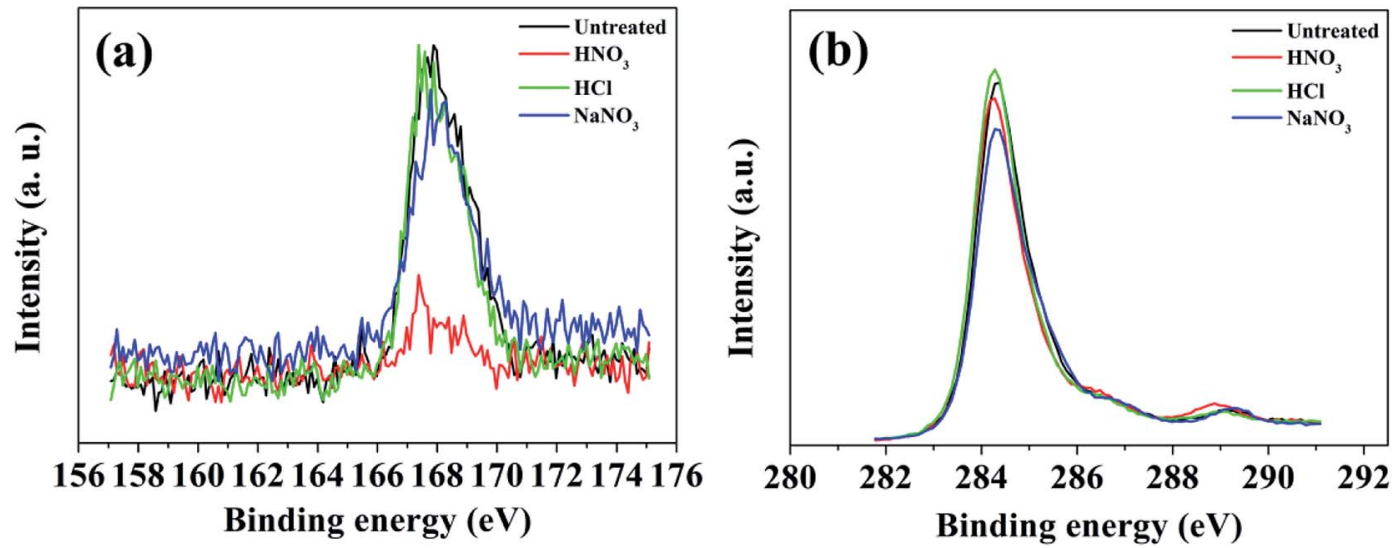

Fig. 6 XPS S2p (a) and C1s (b) spectra of untreated TCFs and TCFs treated by $\mathrm{HNO}_{3}, \mathrm{HCl}$, and $\mathrm{NaNO}_{3}$, respectively. 


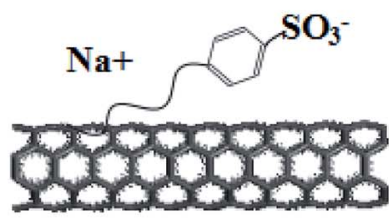

(a) SDBS stick to SWCNTs surface

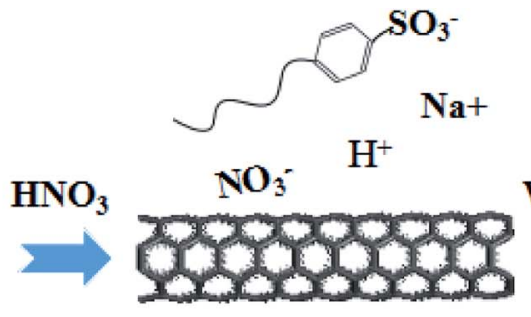

(b) removal of SDBS from SWCNTs

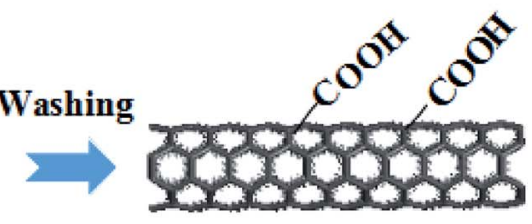

(c) functionalization

Fig. 7 Mechanism of reducing the surface resistance of CNT-TCFs after $\mathrm{HNO}_{3}$ treatment.

treatment with $\mathrm{HNO}_{3}$, which also explains the effectivity of $\mathrm{HNO}_{3}$ treatment. In comparison with the untreated CNT-TCFs, a shift of $2 \mathrm{eV}$ binding energy in the C1s spectra was found in TCFs treated by $\mathrm{HCl}$ and $\mathrm{NaNO}_{3}$, whereas, a shift of $4 \mathrm{eV}$ binding energy in the C1s spectra was found for the $\mathrm{HNO}_{3}$ treated TCFs, as shown in Fig. 6b. This also proved that CNTs underwent a certain extent of chemical oxidation due to $\mathrm{HNO}_{3}$ posttreatment. In general, the $\mathrm{HNO}_{3}$ treatment results in a decrease in the sheet resistance of the CNT-TCFs due to the combined effect of acidity and oxidizability. The strong interaction of the strong acidity and strong oxidizing property of nitric acid causes the SDBS to be removed. At the same time, strong oxidation makes the surface of the CNTs to some extent oxidized.

The Raman and XPS results clearly prove that oxidation happens during the film treatment by $\mathrm{HNO}_{3}$. Therefore, a schematic description of the mechanism is shown in Fig. 7. It is speculated that during the acid treatment process, $\mathrm{NO}_{3}{ }^{-}$and $\mathrm{H}^{+}$in $\mathrm{HNO}_{3}$ remove the SDBS due to their strong interaction with the CNT surface and eventually lead to oxidization. The subsequent water-washing step is to wash away the residual $\mathrm{HNO}_{3}$ and the small amount of SDBS on the CNT-TCFs, hence reducing the contact resistance among the CNTs, resulting in a significant reduction in the sheet resistance of the CNT-TCFs.

\section{Conclusions}

In this study, CNT-TCFs were post-treated by three different reagents for a better understanding of the effect of posttreatment by $\mathrm{HNO}_{3}$, a mechanism of the post-treating process was proposed. By optimizing the experimental conditions, we found that the desired electronic properties of the CNT-TCFs can be achieved under a treatment time of $40 \mathrm{~min}$ and with a ratio of nitric acid to water of $6: 1$. Both SEM and AFM analysis show that the TCFs after being post-treated by $\mathrm{HNO}_{3}$ have a homogenous surface and minimum surface roughness. The comparison studies show that the CNT-TCFs treated by $\mathrm{HNO}_{3}$ possess the best optoelectronic properties with higher transmittance and lower sheet resistance. The reason is because of the oxidation effect and acidity co-existing during posttreatment by $\mathrm{HNO}_{3}$. The strong interaction of the strong acidity and strong oxidizing property of $\mathrm{HNO}_{3}$ causes the SDBS to be removed. At the same time, strong oxidation makes the surface of the CNTs to some extent oxidized. Conclusively, this work reveals that $\mathrm{HNO}_{3}$ treatment can effectively remove the residue of surfactants and improve the CNT-TCFs' properties through an oxidization effect, which significantly advances the understanding in related fields.

\section{Conflicts of interest}

There are no conflicts to declare.

\section{Acknowledgements}

This research was supported by the Science and Technology Plans of Tianjin (No. 15YFXQGX00070), the Science and Technology Plans of Tianjin (No. 15PTSYJC00240), National Natural Science Foundation of China (No. 51378350), and the Nature Science Foundation of Tianjin (No. 17JCTPJC47000).

\section{References}

1 S. Bae, H. Kim, Y. Lee, X. Xu, J. S. Park, Y. Zheng, J. Balakrishnan, T. Lei, H. R. Kim, Y. I. Song, Y. J. Kim, K. S. Kim, B. Özyilmaz, J. H. Ahn, B. H. Hong and S. Iijima, Roll-to-roll production of 30-inch graphene films for transparent electrodes, Nat. Nanotechnol., 2010, 5(8), 574578.

2 F. A. Núria, P. P. Jordi, F. Jordi, I. M. Zahir and R. Siegmar, Flexible, transparent electrodes using carbon nanotubes, Nanoscale Res. Lett., 2012, 7, 571-580.

3 J. H. Park, G.-T. Hwang, S. Kim, J. Seo, H.-J. Park, K. Yu, T.-S. Kim and K. J. Lee, Flash-induced self-limited plasmonic welding of silver nanowire network for transparent flexible energy harvester, Adv. Mater., 2017, 29, 167403.

4 T. K. Sau, A. L. Rogach, F. Jäckel, T. A. Klar and J. Feldmann, Properties and applications of colloidal nonspherical noble metal nanoparticles, Adv. Mater., 2010, 22(16), 1805-1825.

5 D. Choi, M. Lee, H. Kim, W. Chu, D. Chun, S.-H. Ahn and C. S. Lee, Fabrication of transparent conductive tricomposite film for electrochromic application, Appl. Surf. Sci., 2017, 425, 1006-1013.

6 S. Ata, K. Kobashi, M. Yumura and K. Hata, Mechanically durable and highly conductive elastomeric composites from long single-walled carbon nanotubes mimicking the 
chain structure of polymers, Nano Lett., 2012, 12(6), 27102716.

7 A. A. Green and M. C. Hersam, Colored semitransparent conductive coatings consisting of monodisperse metallic single-walled carbon nanotubes, Nano Lett., 2016, 8(5), 1417-1422.

8 Q. Cao, J. Tersoff, D. B. Farmer, Y. Zhu and S. J. Han, Carbon nanotube transistors scaled to a 40-nanometer footprint, Science, 2017, 356(6345), 1369-1372.

9 W. Mao, G. Ai, Y. Dai, Y. Fu, Y. Ma, S. Shi, R. Soe, X. Zhang, D. Qu, Z. Tang and V. S. Battaglia, In situ synthesis of $\mathrm{MnO}_{2} @ \mathrm{CNT}$ microsphere composites with enhanced electrochemical performances for lithium-ion batteries, $J$. Power Sources, 2016, 310, 54-60.

10 N. Mauser, N. Hartmann, M. S. Hofmann, J. Janik, A. Högele and A. Hartschuh, Antenna-enhanced Optoelectronic Probing of Carbon Nanotubes, Nano Lett., 2016, 14(7), 3773-3778.

11 A. R. Inigo, J. M. Underwood and S. R. P. Silva, Carbon nanotube modified electrodes for enhanced brightness in organic light emitting devices, Carbon, 2011, 49(13), 42114217.

12 S. Shi and S. R. P. Silva, High luminance organic lightemitting diodes with efficient multi-walled carbon nanotube hole injectors, Carbon, 2012, 50(11), 4163-4170.

13 L. Hu, D. S. Hecht and G. Grüner, Carbon nanotube thin films: fabrication, properties, and applications, Chem. Rev., 2010, 110(10), 5790-5844.

14 J. Du, S. Pei, L. Ma and H. M. Cheng, 25th anniversary article: carbon nanotube- and graphene-based transparent conductive films for optoelectronic devices, Adv. Mater., 2014, 26(13), 1958-1991.

15 S.-L. Jia, H.-Z. Geng, L.-D. Wang, Y. Tian, C.-X. Xu, P.-P. Shi, Z.-Z. Gu, X.-S. Yuan, L.-C. Jing, Z.-Y. Guo and J. Kong, Carbon nanotube-based flexible electrothermal film heaters with a high heating rate, R. Soc. Open Sci., 2018, 5, 172072.

16 J. Gao, W.-Y. Wang, L.-T. Chen, L.-J. Cui, X.-Y. Hu and H.-Z. Geng, Optimizing processes of dispersant concentration and post-treatments for fabricating singlewalled carbon nanotube transparent conducting films, Appl. Surf. Sci., 2013, 277, 128-133.

17 Y. Tian, X.-C. Zhang, H.-Z. Geng, H.-J. Yang, C.-G. Li, S.-X. Da, X.-S. Lu, J. Wang and S.-L. Jia, Carbon nanotube/ polyurethane films with high transparency, low sheet resistance and strong adhesion for antistatic application, RSC Adv., 2017, 7(83), 53018-53024.

18 U. Dettlaffweglikowska, V. Skákalová, R. Graupner, S. H. Jhang, B. H. Kim, H. J. Lee, L. Ley, Y. W. Park, S. Berber and D. Tománek, Effect of $\mathrm{SOCl}_{2}$ treatment on electrical and mechanical properties of single-wall carbon nanotube networks, J. Am. Chem. Soc., 2005, 127(14), 51255131.

19 S. M. Kim, K. K. Kim, D. L. Duong, Y. Hirana, Y. Tanaka, Y. Niidome, N. Nakashima, J. Kong and Y. H. Lee, Spectroscopic determination of the electrochemical potentials of n-type doped carbon nanotubes, J. Phys. Chem. C, 2012, 116(9), 5444-5449.

20 K. K. Kim, J. J. Bae, H. K. Park, S. M. Kim, H. Z. Geng, K. A. Park, H.-J. Shin, S.-M. Yoon, A. Benayad, J.-Y. Choi and Y. H. Lee, Fermi level engineering of single-walled carbon nanotubes by $\mathrm{AuCl}_{3}$ doping, J. Am. Chem. Soc., 2008, 130(38), 12757-12761.

21 H.-Z. Geng, K. K. Kim, K. P. So, Y. S. Lee, Y. Chang and Y. H. Lee, Effect of acid treatment on carbon nanotubebased flexible transparent conducting films, J. Am. Chem. Soc., 2007, 129(25), 7758-7759.

22 S.-X. Da, J. Wang, H.-Z. Geng, S.-L. Jia, C.-X. Xu, L.-G. Li, P.-P. Shi and G. F. Li, High adhesion transparent conducting films using graphene oxide hybrid carbon nanotubes, Appl. Surf. Sci., 2017, 392, 1117-1125.

23 Z. Zhou, X. Dou, L. Ci, L. Song, D. Liu, Y. Gao, J. Wang, L. Liu, W. Zhou and S. Xie, Temperature dependence of the Raman spectra of individual carbon nanotubes, J. Phys. Chem. B, 2006, 110(3), 1206-1209.

24 Y. Zhang, H. Son, J. Zhang, M. S. Dresselhaus, J. Kong and Z. Liu, Raman spectra variation of partially suspended individual single-walled carbon nanotubes, J. Phys. Chem. C, 2007, 111(5), 1983-1987.

25 U. Dettlaff-Weglikowska, V. Skakalova, J. Meyer, J. Cech, B. G. Mueller and S. Roth, Effect of fluorination on electrical properties of single walled carbon nanotubes and $\mathrm{C}_{60}$ peapods in networks, Curr. Appl. Phys., 2007, 7(1), 42-46.

26 J. Laudenbach, D. Schmid, F. Herziger, F. Hennrich, M. Kappes, M. Muoth, M. Haluska, F. Hof, C. Backes, F. Hauke, A. Hirsch and J. Maultzsch, Diameter dependence of the defect-induced Raman modes in functionalized carbon nanotubes, Carbon, 2017, 112, 1-7. 